\title{
Pseudospectral Methods versus FDTD
}

\author{
Stavros V. Georgakopoulos ${ }^{* 1}$, Constantine A. Balanis ${ }^{1}$, and \\ Rosemary Renaut ${ }^{2}$ \\ ${ }^{1}$ Dept. of Electrical Engineering \\ ${ }^{2}$ Dept. of Mathematics \\ Telecommunications Research Center \\ Arizona State University \\ Arizona State University \\ Tempe, AZ 85287-7206 \\ Tempe, AZ 85287-1804
}

\section{Introduction}

A majority of engineering problems is formulated as a single PDE or a system of PDEs. Usually discrete methods, such as the Finite-Difference (FD) methods, are used to numerically approximate the partial derivatives appearing in the PDEs under consideration. The more accurate the estimation of these derivatives, the more accurate the computed solutions. A new family of methods that can very accurately represent derivatives, and can therefore provide very accurate numerical solutions to PDE problems is the family of Spectral and Pseudospectral Methods $[1],[2]$.

In this paper pseudospectral methods are applied to the solution of electromagnetic problems. Specifically, the Chebychev collocation method is used along with a mapping of the grid to relax the restrictions on the time-step [3]. Entire-domain as well as multi-domain approaches are considered [4]. The system of ODE's that comes from the spectral method formulation is solved using either the standard Runge-Kutta method of order four ( $R K 4$ ), or a newly developed algorithm from the class of diagonally implicit multistage integration methods [5]. The accuracy of the spectral methods for all the different approaches is compared with that of the classical second-order FDTD scheme. Additionally, the CPU time as well as the memory required to achieve certain accuracy are reported for both the spectral and the FDTD methods.

\section{Formulation \& Results}

Initially, the one-dimensional (1-D) Maxwell's equations for the $T M^{z}$ mode are examined. The domain considered here is a free-space domain one wavelength long $(\ell=\lambda)$ at $300 \mathrm{MHz}$. It is excited at the left boundary with a Gaussian pulse that has significant frequency components up to $1.5 \mathrm{GHz}$ ( $10 \%$ bandwidth) and is terminated at the right boundary using the exact solution. The simulation time is chosen to be $t_{s}=0.9 \mathrm{l} / \mathrm{c}$, where $c$ is the speed of light. For all the different approaches that are implemented the error is computed in the maximum norm at time $t=t_{s}$.

Here, some new ways of evaluating derivatives, using spectral methods, are introduced. The idea of differentiation matrices comes from interpolation theory. Assume that the data $u_{i}=u\left(x_{i}\right), i=0,1, \ldots, N$, represent the values of a function $u(x)$ whose first derivative is to be computed at the points $x_{i}, u^{\prime}\left(x_{i}\right)$. Assuming that the function has a sufficient degree of smoothness we find the polynomial $p_{N}(x)$ that interpolates $u(x)$ at the grid points so that $p_{N}\left(x_{i}\right)=u\left(x_{i}\right)$. 
Based on Lagrange interpolation the polynomial $p_{N}(x)$ can be written as $p_{N}(x)=$ $\sum_{i=0}^{N} u_{i} \ell_{i}(x)$, where $\ell_{i}(x)$, are the $N^{\text {th }}$ degree Lagrange interpolating polynomials. The first derivative of the function $u(x)$ can be computed using the interpolant polynomial $u^{\prime}\left(x_{j}\right) \simeq p_{N}^{\prime}\left(x_{j}\right)=\sum_{i=0}^{N} u_{i} \ell_{i}^{\prime}\left(x_{j}\right)=\sum_{i=0}^{N} u_{i} d_{j i}$, where $d_{j i}=\ell_{i}^{\prime}\left(x_{j}\right)$. This can be reformulated in a matrix form if we set $\underline{u}=\left[u_{0}, u_{1}, u_{2}, \ldots, u_{N}\right]^{T}, \underline{v}=$ $\left[p_{N}^{\prime}\left(x_{0}\right), p_{N}^{\prime}\left(x_{1}\right), p_{N}^{\prime}\left(x_{2}\right), \ldots, p_{N}^{\prime}\left(x_{N}\right)\right]^{T}$, and $D_{j i}=d_{j i}$, as $\underline{v}=D \underline{u}$, where $D$ is called differentiation matrix (DM). Obviously, in order to compute the elements of $D$ the values of $\ell_{i}^{\prime}\left(x_{j}\right)$ should be evaluated [2].

Direct application of the entire-domain pseudospectral collocation method is quite straightforward. The spatial derivatives are approximated by using the differentiation matrices (DM) and an appropriate grid. Assuming a Chebychev grid $x_{i}, i=0,1, \ldots, N$, and assuming grid values for the fields $E_{z_{i}}$ and $H_{y_{i}}$ we can write the semi-discrete form of Maxwell's equations as:

$$
\frac{\partial \underline{H}_{y}}{\partial t}=\frac{1}{\mu}\left(D \underline{E}_{z}-\rho \underline{H}_{y}\right), \quad \frac{\partial \underline{E}_{z}}{\partial t}=\frac{1}{\epsilon}\left(D \underline{H}_{y}-\sigma \underline{E}_{z}\right)
$$

where $\underline{H}_{y}$ and $\underline{E}_{z}$ are the vectors containing the grid values of the field components and $D$ the differentiation matrix. The Chebychev grid on the interval $[-1,1]$ is formed by the extrema of the $N^{\text {th }}$ degree Chebychev polynomial $T_{N}(x)$ [roots of the first derivative of $\left.T_{N}(x), T_{N}^{\prime}(x)\right]$, which are given by $x_{j}=-\cos (\pi j / N), j=0,1, \ldots, N$. Also, the Chebychev grid can be mapped to a different grid following the technique proposed in [3] in order to relax the time-step restrictions for stability. Notice that the mapping is controlled by a parameter $j$, where $j=N / 2$ corresponds to the Chebychev grid and as $j$ decreases the maximum time-step allowed for stability increases. Furthermore, it has been shown that by choosing $j=N / 2 \pi$ the exponential convergence of the spectral methods is retained. Figure 1 illustrates the errors of the entire domain collocation method for the standard Chebychev grid as well as the mapped ones, and compares them with the error of the standard second-order FDTD. It should be pointed out that the time-step used in FDTD was chosen as $\Delta t=0.5 \Delta x / c$. Obviously, the spectral method for all different grids outperforms the FDTD method in terms of accuracy. It is quite impressive that in order to get the same accuracy as that of the spectral Chebychev method with a computational domain of 50 points, an FDTD domain of 8000 points is needed. Therefore, by using spectral methods, high accuracy can be achieved without very fine spatial discretization. The main drawback though of spectral methods is that they exhibit very restrictive stability criteria, forcing us to discretize very finely in time. However, this can be resolved using mapped grids which relax the time-step restrictions and reduce the CPU time as shown in Figure 1. Furthermore, Figure 2 shows the CPU time versus the maximum error for all different methods.

Besides the entire domain formulation, multi-domain approaches are also examined here. Multi-domain approaches have the advantage of using smaller differentiation matrices and a larger time-step because each sub-domain is formed by a number of points considerably smaller than the one of the entire domain method. There are two main multi-domain formulations used to patch the sub-domains: (a) the method of characteristics and (b) the fictitious points approach [4]. The characteristic methods are divided into two categories: correctional and differential. Here, due to space limitations only results corresponding to characteristic methods are shown. However, in the presentation the fictitious points approach will also be considered. The 
1-D problem already examined above is solved again using the correctional and differential multi-domain approaches. The maximum error for different number of sub-domains is plotted versus the total number of points used in the sub-domains for the correctional approach in Figure 3. Moreover, the same figure shows the CPU time versus the maximum error. Similar graphs are illustrated in Figure 4 for the differential approach. It can be concluded that, for the problem examined here, the two multi-domain methods, correctional and differential, exhibit the same accuracy

In this manuscript only 1-D problems have been analyzed. However, 2-D formulations are under implementation and will be presented in the conference. Also, notice that the system of ODE's, coming from the spectral methods formulation, has been solved using the standard Runge-Kutta of order four (RK4). However, the use of newly developed algorithms such as the diagonally implicit multistage integration methods [5] is being investigated and the results will be shown in the presentation.

\section{References}

[1] C. Canuto, M. Y. Hussajni, A. Quarteroni, and T. Zang, Spectral Methods in Fluid Dynamics. New York: Springer-Verlag, 1988

[2] B. Fornberg, A Practical Guide to Pseudospectral Methods. Cambridge: Cambridge University Press, 1996.

[3] D. Kosloff and H. Tal-Ezer, "A modified pseudospectral method with an $O\left(N^{-1}\right)$ time step restriction," J. Comput. Phys., vol. 104, pp. 457-469, 1993.

[4] T. A. Driscoll and B. Fornberg, "Block pseudospectral method for Maxwell's equations II: Two dimensional case, discontinuous coefficient case.," SIAM J. Sci. Comput, vol. 21, pp. 1146-1167, 1999.

[5] J. C. Butcher and Z. Jackiewicz, "Implementation of diagonally implicit general multistage integration methods for ordinary differential equations," SIAM J. Numer, Anal, vol. 34, pp. 2119-2141, 1997.
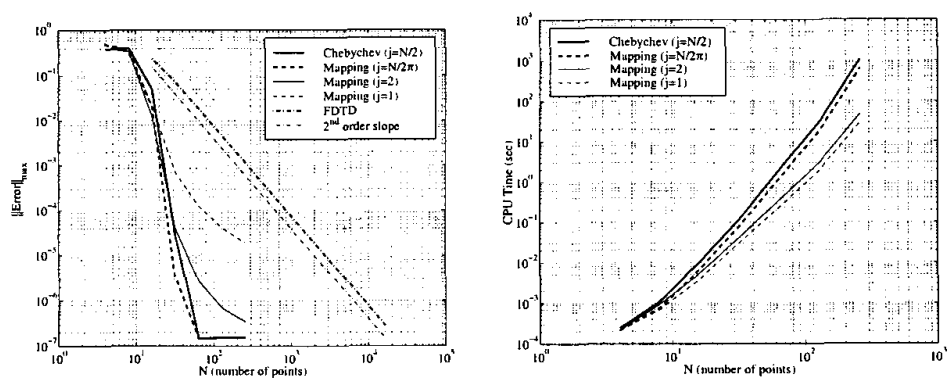

Figure 1: Results from the entire domain approach 


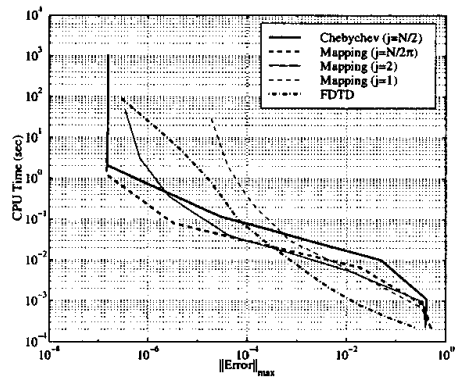

Figure 2: CPU time versus error for the entire domain approach.
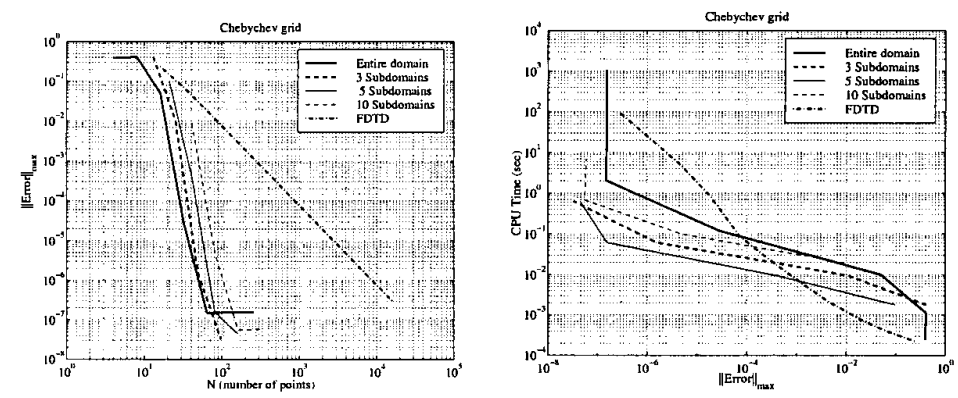

Figure 3: Results from the multi-domain correctional approach.
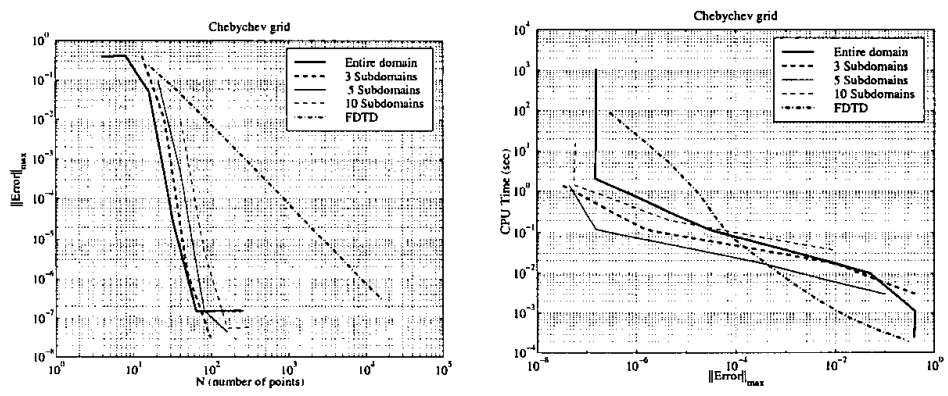

Figure 4: Results from the multi-domain differential approach. 